\title{
Intracardiac acoustic radiation force impulse imaging: A novel imaging method for intraprocedural evaluation of radiofrequency ablation lesions
}

\author{
Stephanie A. Eyerly, MS, ${ }^{*}$ Tristram D. Bahnson, MD, FHRS, ${ }^{\dagger}$ Jason I. Koontz, MD, PhD, ${ }^{\dagger}$ \\ David P. Bradway, BS, ${ }^{\ddagger}$ Douglas M. Dumont, PhD ${ }^{\ddagger}$ Gregg E. Trahey, PhD, ${ }^{\star \ddagger}$ Patrick D. Wolf, PhD ${ }^{\ddagger}$ \\ From the *Department of Biomedical Engineering, Duke University, ${ }^{\dagger}$ Duke Center for Atrial Fibrillation, Duke Heart \\ Center, and Clinical Cardiac Electrophysiology Section, Division of Cardiovascular Medicine, Duke University, and \\ *Department of Radiology, Duke University Medical Center, Durham, North Carolina.
}

BACKGROUND Arrhythmia recurrence after cardiac radiofrequency ablation (RFA) for atrial fibrillation has been linked to conduction through discontinuous lesion lines. Intraprocedural visualization and corrective ablation of lesion line discontinuities could decrease postprocedure atrial fibrillation recurrence. Intracardiac acoustic radiation force impulse (ARFI) imaging is a new imaging technique that visualizes RFA lesions by mapping the relative elasticity contrast between compliant-unablated and stiff RFA-treated myocardium.

OBJECTIVE To determine whether intraprocedure ARFI images can identify RFA-treated myocardium in vivo.

METHODS In 8 canines, an electroanatomical mapping-guided intracardiac echo catheter was used to acquire 2-dimensional ARFI images along right atrial ablation lines before and after RFA. ARFI images were acquired during diastole with the myocardium positioned at the ARFI focus $(1.5 \mathrm{~cm})$ and parallel to the intracardiac echo transducer for maximal and uniform energy delivery to the tissue. Three reviewers categorized each ARFI image as depicting no lesion, noncontiguous lesion, or contiguous lesion. For comparison, 3 separate reviewers confirmed RFA lesion presence and contiguity on the basis of functional conduction block at the imaging plane location on electroanatomical activation maps.

RESULTS Ten percent of ARFI images were discarded because of motion artifacts. Reviewers of the ARFI images detected RFAtreated sites with high sensitivity (95.7\%) and specificity (91.5\%). Reviewer identification of contiguous lesions had $75.3 \%$ specificity and $47.1 \%$ sensitivity.

CONCLUSIONS Intracardiac ARFI imaging was successful in identifying endocardial RFA treatment when specific imaging conditions were maintained. Further advances in ARFI imaging technology would facilitate a wider range of imaging opportunities for clinical lesion evaluation.

KEYWORDS ARFI imaging; Radiofrequency ablation; Lesion assessment; Atrial fibrillation

ABBREVIATIONS 2-D = 2-dimensional; $\mathbf{A R F I}=$ acoustic radiation force impulse; DOF = depth of field; EAM = electroanatomical mapping; ECG = electrocardiogram; ICE = intracardiac echo; LAT = local activation time; $\mathbf{R F A}=$ radiofrequency ablation; TCA $=$ transcatheter cardiac ablation

(Heart Rhythm 2012;9:1855-1862) ๑ 2012 Heart Rhythm Society. All rights reserved.

\section{Introduction}

Radiofrequency ablation (RFA) is an established curative therapy for cardiac arrhythmias. ${ }^{1}$ Transcatheter ablation (TCA) for atrial fibrillation electrically isolates arrhythmogenic regions of the atria by creating contiguous and trans-

This study was funded by the National Institutes of Health (grant numbers R01-EB-012484, R21-EB-007741, and R37-HL-096023). This study used loaned equipment from Siemens Healthcare (ACUSON S2000 ultrasound scanner: Issaquah, WA) and Biosense Webster, Inc (CARTO XP EP Navigation System: Diamond Bar, CA). Dr Trahey reports relevant patent application and ownership. Address for reprint requests and correspondence: Dr Patrick D. Wolf, PhD, Department of Biomedical Engineering, Duke University, 136 Hudson Hall, PO Box 90281, Durham, NC 27708. E-mail address: patrick.wolf@ duke.edu. mural lines of multiple discrete lesions with radiofrequency energy. Therefore, lesion placement and line contiguity are important determinants of procedure efficacy. ${ }^{1-3}$

To date, there is no clinically proven method to visually evaluate the presence or extent of RFA lesions during TCA. Electroanatomical mapping (EAM) systems guide lesion placement, annotate the catheter position during RF energy delivery, and construct substrate conduction maps. EAM conduction maps provide indirect feedback about lesion formation during RFA, but they cannot provide direct lesion visualization. Fluoroscopy and intracardiac ultrasound imaging (intracardiac echo [ICE]) can guide catheter placement during TCA, but they lack the soft-tissue contrast needed to provide lesion assessment. ${ }^{4}$ Intraprocedural visualization of RFA lesions could improve TCA outcomes by identifying in- 
complete RFA lesion formation and guiding additional ablation to complete the lesion set.

Acoustic radiation force impulse (ARFI) imaging is a novel ultrasound-based technique that creates 2-dimensional (2D) images of relative tissue elasticity. ${ }^{5}$ ARFI imaging uses ultrasonic radiation force impulses to mechanically displace tissue and conventional ultrasound methods to monitor the tissue response spatially and temporally. The displacement magnitude $(\sim 10 \mu \mathrm{m})$ is calculated by using correlation-based delay estimation methods. ${ }^{6,7}$ The magnitude of the tissue displacement is inversely proportional to tissue elasticity, and a 2D image of ARFI-induced tissue displacement provides visualization of relative tissue stiffness. ${ }^{8,9}$

RF-induced tissue heating causes irreversible thermocoagulation and permanently denatures intracellular and contractile proteins, which increases tissue stiffness. ${ }^{10-12} \mathrm{We}$ have previously demonstrated that ICE-based ARFI imaging can visualize the relative elasticity difference between ablated and unablated myocardium in vivo and can accurately assess focal RFA lesion morphology in vitro. ${ }^{13-15}$

As currently implemented, 2D ICE-based ARFI images can be acquired and displayed every few seconds; this frame rate makes it difficult to "scan" the heart for lesions. ICEbased ARFI imaging can be integrated with EAM to guide the ARFI imaging plane to RFA annotated areas, enabling efficient intraprocedure lesion evaluation.

This animal study was undertaken to determine whether intracardiac ARFI imaging can be used to detect RFA treatment and evaluate conduction block-producing contiguous lesion lines during in vivo TCA.

\section{Methods}

\section{Integrated imaging system and procedural equipment setup}

The multimodality imaging system consisted of a modified CARTO XP EP Navigation System (Biosense Webster, Diamond Bar, CA) and a software-modified Siemens ACUSON S2000 ultrasound scanner (Siemens Healthcare, Issaquah, WA). A custom CARTOSound module (Biosense Webster; Software Development, Tirat Carmel, Israel) was installed to display the S2000 ICE image in the CARTO XP software. Conventional ICE and ARFI images were acquired with a commercially available 10-F SoundStar catheter without modification (Biosense Webster). ARFI images were acquired and displayed on a laptop computer (Dell Precision M90) within 15 seconds during the procedure.

Catheters were navigated to the heart with fluoroscopy (C-Arm, Philips Healthcare, Andover, MA). All focal RFA lesions were delivered with a Stockert 70 Generator and NaviStar mapping/ablation catheter (Biosense Webster) with a peak energy of 30-45 W under temperature control with a target temperature between $50^{\circ} \mathrm{C}$ and $60^{\circ} \mathrm{C}$ for $\sim 30-90$ seconds. An octapolar sensing catheter (XT Steerable, Bard, Lowell, MA) was positioned in the coronary sinus, and the distal electrodes were used as the activation mapping reference and pacing electrodes (S48 Square Pulse Stimulator, Grass Technologies, West Warwick, RI).

The surface electrocardiogram (ECG) and endocardial electrograms from the sensing and mapping catheters were acquired by the CARTO patient interface unit, input into an Octal Bio Amp (ADInstruments, Milford, MA), and recorded by using LabChart 7.0 data acquisition software (PowerLab, ADInstruments). In LabChart, an output channel was configured to provide an impulse at a user-selected delay after a detected QRS of the ECG. This signal was fed into the ultrasound scanner as a pseudo-ECG and used to gate the image acquisitions.

\section{Animal experiment procedure}

The animal study protocol was approved by the Duke University Animal Care and Use Committee and conformed to the Guide for the Care and Use of Laboratory animals. ${ }^{16}$

Eight canine subjects were anesthetized (preanesthesia: intramuscular acepromazine $0.02-0.05 \mathrm{mg} / \mathrm{kg}$ followed by intravenous propofol $4-6 \mathrm{mg} / \mathrm{kg}$; general anesthesia: isoflurane gas $1 \%-5 \%$ via inhalation), intubated, ventilated, and maintained metabolically stable. A baseline point-bypoint geometry and local activation time (LAT) map was made of the canine right atrium. A pre-RFA guideline of points delineating the intended sites of ablation was drawn on the CARTO LAT map; baseline ARFI images were acquired along this guideline. The ICE fan position and orientation for each ARFI imaging location was saved for postprocedure review using the navigation features of the EAM system. Discrete RFA lesions were delivered along the guideline in 2 stages. The first stage left an intentional approximately $1-\mathrm{cm}$ gap, and the gap was closed in the second stage. After each stage, a new LAT map was constructed and the ablation line was ARFI imaged. At the end of the study, the animal was euthanized and the heart was removed to confirm lesion delivery. The RFA lesions were photographed with a digital camera.

\section{In vivo ARFI imaging implementation}

The ARFI images were created using a standard 64-element SoundStar catheter imaging, at $6.15 \mathrm{MHz}$ (1.5-cm focus). The S2000 ultrasound scanner was software-modified to implement $2 \mathrm{D}$ beam sequences that acquire spatially and temporally registered B-mode and ARFI images. A single ARFI image covered a 37-degree field of view and required approximately $92 \mathrm{~ms}$ to acquire. Image data acquisition, data transfer, data processing, and image display took approximately 10-15 seconds per ARFI image. All imaging sequences used were within regulatory limits for mechanical index $(<1.7)$ and transducer surface heating $\left(<4.0^{\circ} \mathrm{C}\right) .{ }^{17}$

In this study, ARFI images were acquired with the myocardium positioned near the ARFI imaging focus $(1.5 \mathrm{~cm})$ and parallel to the ICE transducer. ARFI imaging of the myocardium outside the appropriate imaging depth of field (DOF; between 0.5 and $2.25 \mathrm{~cm}$ ) substantially reduces the acoustic energy delivered to the tissue and subsequently the relative elasticity contrast. ${ }^{14}$ ARFI imaging the endocardial 
surface at an oblique angle creates nonuniform acoustic energy delivery into the tissue, resulting in depth-dependent differences in relative tissue displacement. Any ARFI images where the myocardial surface was outside the DOF or at an oblique angle ( $>50$ degree) were discarded. Last, ARFI images with the mapping catheter in the field of view were excluded from analysis because the catheter tip blocked acoustic energy from entering the tissue, creating a shadowing artifact.

All ARFI acquisitions in this experiment were also ECGgated and acquired during diastole by using the programmable LabChart output channel. ARFI imaging during diastole reduces artifacts caused by cardiac motion; bulk motion of the myocardium during the acquisition can corrupt the tissue displacement measurements. ${ }^{13,14}$ Acquisitions were also gated to diastole to maximize the stiffness contrast between RFA lesions and the surrounding unablated myocardium. ${ }^{13,14,18}$ To demonstrate the elasticity contrast between systole and diastole in ARFI imaging, Figure 1A presents ARFI images (acquired with a VF10-5 linear transducer, Siemens Healthcare) of a ventricular epicardial RFA lesion at different times in the cardiac cycle. The lesion is visible as a semicircular region of relatively low ARFI-induced displacement. The ARFI image stiffness contrast between the lesion and unablated myocardium is maximal during diastole (Figures 1A-1 and 1A-4). The larger 128-element ultrasound transducer was used for this demonstration because it allowed a fast imaging frame rate $(14 \mathrm{~Hz})$ to observe the stiffening of the myocardium. Frame rates this high damage existing intracardiac probes.

\section{ARFI image processing}

The 2D ARFI images were formed by delivering acoustic impulses to the tissue and tracking the resulting displacement for approximately $1 \mathrm{~ms}$ at each pixel by using the normalized cross-correlation methods outlined by Hsu et al. ${ }^{14}$ Regions of the image with poor displacement estimates (blood, tissue beyond the DOF) were removed by thresholding the median normalized cross-correlation coefficient. Pixel locations with a large displacement standard deviation $(>1 \mu \mathrm{m})$ through the tracking period were also removed. A quadratic extrapolation motion filter was applied to the raw ARFI image data to reduce the effects of bulk physiological motion and catheter rebound on the displacement estimates. ${ }^{7,14}$ The maximum ARFI-induced displacement values for each pixel location throughout the tracking time were combined to create a single $2 \mathrm{D}$ maximum ARFIinduced displacement image. ARFI images that exhibited substantial motion artifacts such as low normalized crosscorrelation coefficients within the myocardium $(<0.75)$ or visible myocardial discontinuity were not included in the final statistical analysis.

For the final display, the upper limit of the ARFI displacement color bar was initially set to $10 \mu \mathrm{m}$ and manually
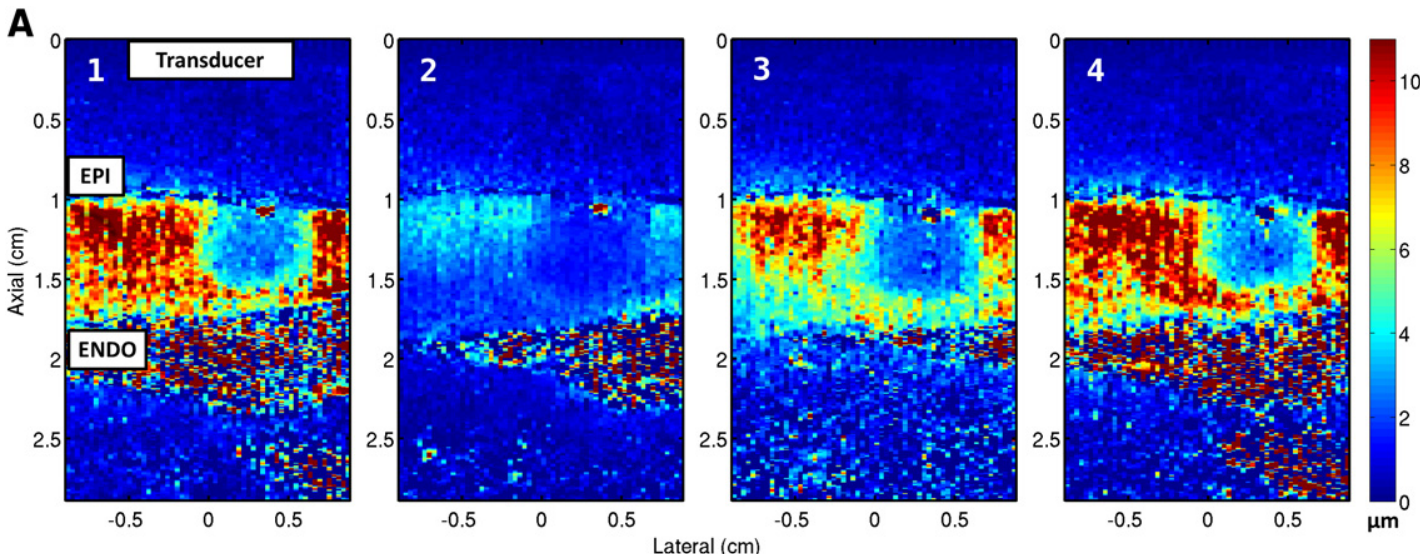

B

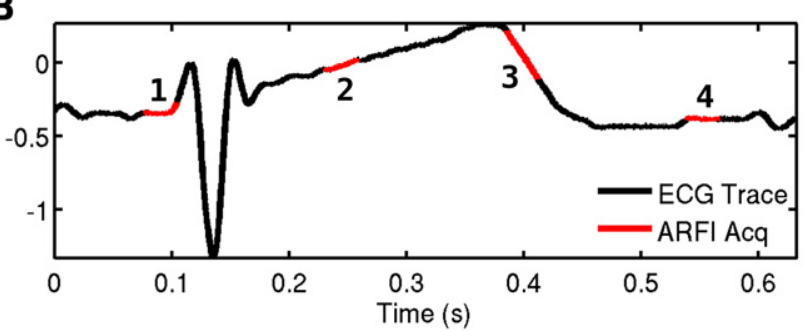

Figure 1 ARFI images of a right ventricular epicardial RFA lesion through the cardiac cycle. Ablation catheter plane was vertically parallel to the page; the nonirrigated electrode tip ablated the epicardial surface (EPI = epicardium, ENDO = endocardium) for $7 \mathrm{~s}$ at $20 \mathrm{~W}$. A: ARFI images acquired at different times during the cardiac cycle: (1) and (4) during diastole, (2) during systole, and (3) during end systole. ARFI images were acquired with a linear transthoracic probe (VF10-5, Siemens Medical) vacuum suctioned on the RV epicardium and imaging through a standoff. Color bar units are maximum displacement away from the transducer in microns; the transducer face is the top edge in the figure. Within the lesion, the ARFI-induced displacement is small (blue) throughout the cycle. The normal myocardium cycles between high displacement (red) during diastole and low displacement during systole. B: ARFI acquisition times corresponding to the ECG. ARFI = acoustic radiation force impulse; ECG = electrocardiogram; RFA $=$ radiofrequency ablation; RV = right ventricle. 
adjusted to increase contrast and reduce saturation. The lower limit of the color bar was always set to $0 \mu \mathrm{m}$. Energy delivery was maximal at the focus, and the color bar range was typically increased for images where the myocardium was directly at the focus and decreased when it was located above or below the focus. The range for all images of the upper limit of the color bar was 7.5-15 $\mu \mathrm{m}$.

\section{EAM LAT maps}

Screenshots of each imaging fan position were compiled with the LAT map by using the CARTOSound Module in the system Review Mode. The tricuspid annulus was cut from the map, and internal points were removed. The ablation and guideline markers were hidden, and the color scale of each LAT map was adjusted to increase the color gradient at the ablation line.

\section{Image review and statistical analysis}

The ARFI images and their corresponding LAT maps were separately randomized and each read by 3 different reviewers (6 total reviewers).

Three reviewers (all experienced in ARFI imaging) were instructed to classify the ARFI images into 1 of 3 categories: (1) no lesion, defined as the ARFI image depicts no visible area of low relative displacement in the myocardium; (2) noncontiguous lesion, depicts at least 1 area of low relative displacement that does not extend the entire length or depth of the imaged myocardium; or (3) contiguous lesion, the full extent of the imaged myocardium depicts low relative displacement.

Three different reviewers ( 2 clinical electrophysiologists and 1 electrophysiology expert) were posed 3 yes or no questions about each LAT map in reference to the baseline map: (1) "Is there conduction block anywhere on this LAT map?" (additional possible answer of "Cannot be determined"); (2) "Based on the activation pattern in the LAT map, does there appear to be RF lesions present at the ICE imaging fan location?"; and (3) "Is the ICE imaging plane completely parallel to and transecting a line of block, thereby imaging a continuous line of lesion?" The RFA lesion widths observed in the postmortem pathology photographs were typically between 4 and $8 \mathrm{~mm}$; therefore, questions 2 and 3 were answered considering the imaging plane could be transecting RFA lesions within approximately $8 \mathrm{~mm}$ of conduction block.

The degree of agreement within the sets of 3 reviewers was quantified with the kappa statistic. ${ }^{19}$ The majority (at least 2 of the 3 reviewers) reviews for both the ARFI images and the LAT maps were used to determine the final imagemap pair assessment. Image pairs where the LAT-map assessment had no majority review or the majority review misidentified the correct stage of ablation (question 1; preRFA vs post-RFA) were also removed from the analysis.

Heterogeneity analysis was performed to justify pooling the data for all animals. A heterogeneity chi-square statistic was calculated to confirm that the data from the different animals came from a homogeneous population. ${ }^{20}$
The majority ARFI image categorization and LAT-map assessment from question 2 were used to compile a $2 \times 2$ contingency table of all image pairs to summarize the ability of ARFI imaging to identify the RFA lesion. For the ARFI images, the category "Lesion" included the noncontiguous and continuous classifications. A second $2 \times 2$ contingency table was compiled to summarize the ability of ARFI imaging to identify lesion line discontinuities and conduction block by using the LAT-map assessments from questions 2 and 3. This table only included postablation image pairs with a yes response to LAT-map assessment question 2 . The sensitivity, specificity, positive predictive value, and negative predictive value were calculated for both tables.

\section{Results}

In 8 canine subjects, 243 ARFI images were acquired with the outlined imaging conditions. Twenty-four images were rejected because of motion artifact; the remaining 219 ARFI image-LAT map pairs (54 preablation, 80 after incomplete ablation, and 85 after gap closure) were reviewed for statistical analysis. The kappa coefficient for the ARFI image assessments indicated substantial agreement $(\kappa=0.734)$ between the 3 reviewers. The individual kappa coefficients for the LAT-map review questions showed almost perfect agreement when reading for global conduction block $(\kappa=$ 0.909), substantial agreement for identifying lesions at the imaging plane $(\kappa=0.684)$, and moderate agreement when assessing full conduction block at the imaging plane $(\kappa=$ 0.428). The LAT-map reviewers had different question responses (no majority review) for 15 image pairs. The majority review misidentified the correct stage of ablation in 5 LAT maps. These 20 image pairs were excluded from the final analysis.

The statistical calculations included 199 image pairs. The reviews for detecting RFA treatment with ARFI imaging compared with the reviews for identifying conduction disturbance in the LAT map are shown in Table 1. Table 2 shows the ARFI image reviews for RFA lesion contiguity vs functional conduction block ( $\mathrm{n}=140$, includes only postablation image pairs confirmed by the LAT-map review). The heterogeneity analysis justified pooling the data from all the animal subjects. Table 3 summarizes the heterogeneity chisquare and $P$ value, sensitivity, specificity, positive predictive value, and negative predictive value for Tables 1 and 2 .

Table 1 ARFI image assessments for RFA treatment

LAT map assessment at imaging fan location

\begin{tabular}{lllc}
$\begin{array}{l}\text { ARFI image } \\
\text { lesion } \\
\text { assessment }\end{array}$ & $\begin{array}{l}\text { No conduction } \\
\text { disturbance }\end{array}$ & $\begin{array}{l}\text { Conduction disturbance } \\
\text { inferring the presence } \\
\text { of lesions }\end{array}$ & Total \\
\hline No lesion & 54 & 6 & 60 \\
Lesion & 5 & 134 & 139 \\
Total & 59 & 140 & 199 \\
\hline
\end{tabular}

ARFI $=$ acoustic radiation force impulse; LAT = local activation time; $\mathrm{RFA}=$ radiofrequency ablation. 
Table 2 ARFI image assessments for contiguous RFA

LAT map assessment at imaging fan location

\begin{tabular}{lllr} 
ARFI image lesion assessment & No block & Block & Total \\
\hline Noncontiguous lesion & 67 & 27 & 94 \\
Contiguous lesion & 22 & 24 & 46 \\
Total & 89 & 51 & 140 \\
\hline
\end{tabular}

ARFI = acoustic radiation force impulse; LAT = local activation time; RFA $=$ radiofrequency ablation.

An image pair example from each ablation stage is shown in Figure 2. Before RFA (Figure 2C-1) the myocardial elasticity is homogeneous with relatively high ARFIinduced displacements. Figure 2C-2 shows the unablated gap in the lesion line, visible as the area of high tissue displacement $(>7.5 \mu \mathrm{m})$ surrounded by areas of relatively low tissue displacements $(<4.5 \mu \mathrm{m})$. The final ARFI image (Figure 2C-3) shows a homogeneous region of low displacement, confirming complete ablation of the gap.

\section{Discussion}

This study demonstrated that ARFI imaging, a new ultrasound-based elasticity imaging technique, has the potential to provide intraprocedure visualization of endocardial RFA lesions during TCA. RFA lesions were identified as regions with low ARFI-induced tissue displacement, indicating increased stiffness, in contrast to the relatively high displacement of unablated myocardium. Conduction disturbances at the imaging site on the LAT maps confirmed RFA treatment. Reviewers of the ARFI images accurately detected RFA-treated areas from nontreated areas with high sensitivity, specificity, and predictive values. Reviewers also distinguished unablated gaps between adjacent RFA lesions that corresponded to conduction "breakthrough" on the activation map. Furthermore, ARFI images reviewed as contiguous RFA frequently displayed conduction block on the activation map. Overall, we believe that using intracardiac ARFI imaging for intraprocedural elasticity-based lesion evaluation is feasible and merits further investigation.

\section{Clinical translation and implications}

Lesion evaluation with an ARFI imaging EAM system could guide the delivery of consolidating RFA to lesion line discontinuities and subsequently decrease the total procedure time and increase the success rate of procedures that use linear ablation strategies, such as pulmonary vein iso- lation. An ARFI imaging EAM system uses existing clinical tools and techniques; therefore, clinics would not need to purchase and install expensive new equipment in the operating rooms and electrophysiologists would not need significant training to use the system.

As implemented in this study, the primary difficulty for translating intracardiac ARFI imaging to clinical practice is the specific imaging conditions required to create usable ARFI images. The ICE catheter must be within $2 \mathrm{~cm}$ of the myocardium or the tissue cannot be detectably displaced by the acoustic impulse, and the endocardial surface must be nearly parallel to the transducer or the uneven distribution of radiation force leads to spatial differences in displacement unrelated to the relative tissue elasticity. Future work to prepare ARFI imaging for widespread clinical adoption could include the production of an ICE catheter with a transducer optimized for ARFI imaging to increase the imaging DOF and developments in ARFI imaging sequencing and image processing methods for energy-depth normalization when imaging curvilinear surfaces. Also, current intracardiac ARFI imaging technology limits the imaging rate to 3 or 4 acquisitions/min. Improvements for near real-time imaging will have to balance increasing acquisition rates against increasing acoustic exposure (mechanical index/transducer face heating).

Finally, imaging was gated to diastole to minimize motion artifacts, and the ARFI-induced displacement data were motion filtered to reduce the effects of axial bulk motion on the displacement estimations. Unfortunately, as seen in approximately $10 \%$ of the acquired images in this study, motion artifacts can occur if the acquisition is mistimed because of changes in heart rate or if the tissue motion is perpendicular to the imaging plane. Experience and the development of more sophisticated gating techniques could reduce the incidence of motion artifacts in the images. Also, the canine heart rates (often $>120$ beats/min) were higher than those of typical human patients; slower heart rates provide a longer diastolic window for imaging, subsequently reducing the frequency of mistimed acquisitions. However, it is unknown how the inability to ECG-gate imaging during atrial fibrillation will affect ARFI image quality and contrast. Despite the limitations, a substantial number of high-quality images of ablation lesions were obtained in vivo and some clinicians may find utility in the technique even without significant advances in the imaging technology.

Table 3 Summary of statistical analysis for Tables 1 and 2

\begin{tabular}{llllllll}
\hline & $\mathrm{n}$ & Heterogeneity $x^{2}$ & $\begin{array}{l}\text { Heterogeneity } \\
P \text { value }^{*}\end{array}$ & Sensitivity (\%) & Specificity (\%) & PPV (\%) & NPV (\%) \\
\hline RFA treatment (1) & 199 & 4.59 & .7099 & 95.7 & 91.5 & 96.4 & 90.0 \\
Contiguous RFA (2) & 140 & 9.29 & .2325 & 47.1 & 75.3 & 52.2 & 71.2 \\
\hline
\end{tabular}

$\mathrm{NPV}=$ negative predictive value; PPV = positive predictive value; RFA = radiofrequency ablation.

*Heterogeneity analysis: Degrees of freedom $=7$, do not reject $H_{0}$ (8 samples are from a homogeneous population). 
A
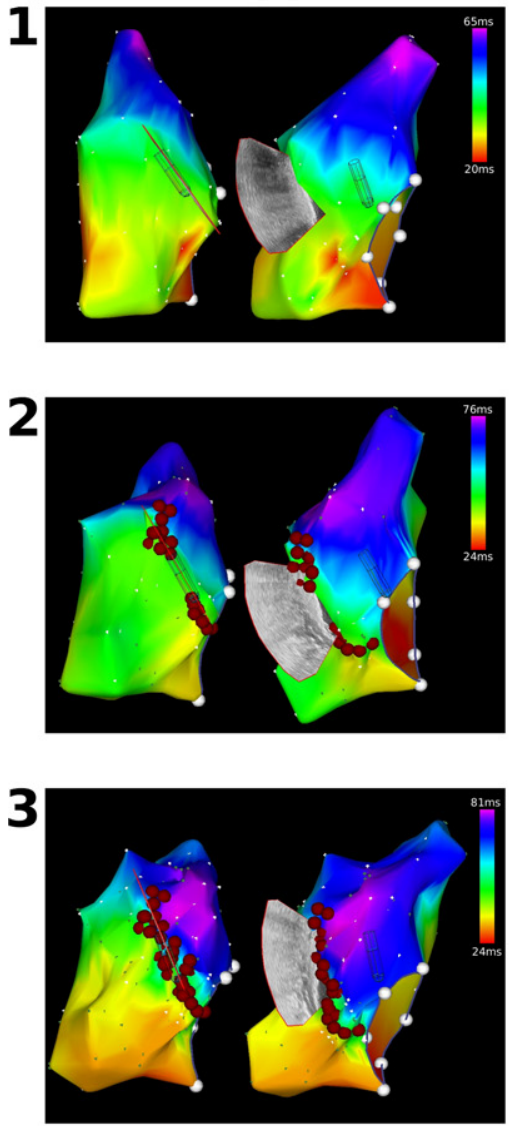

B
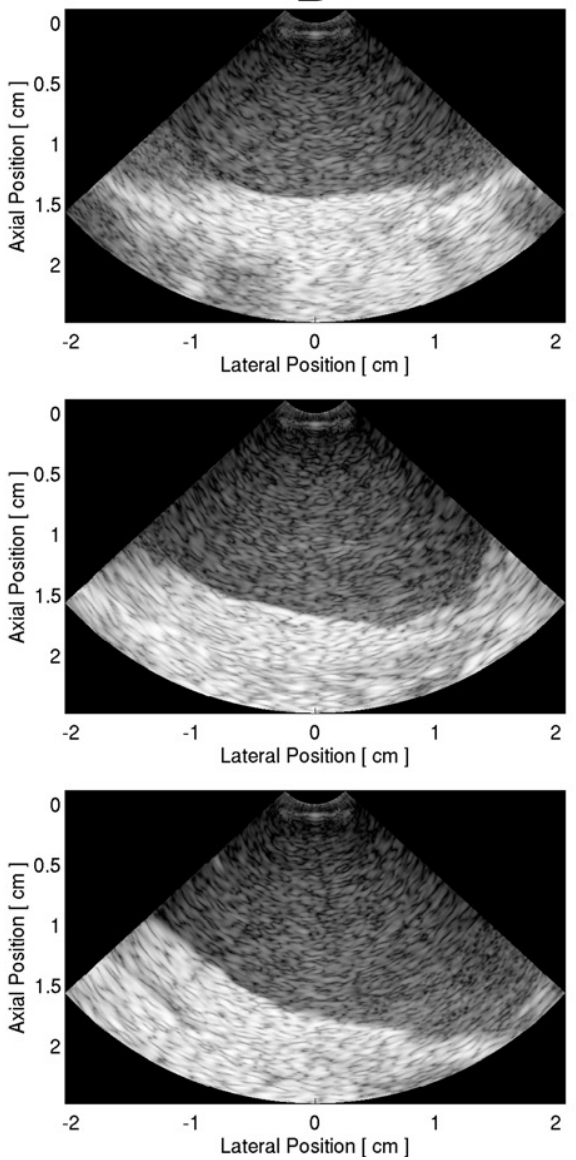
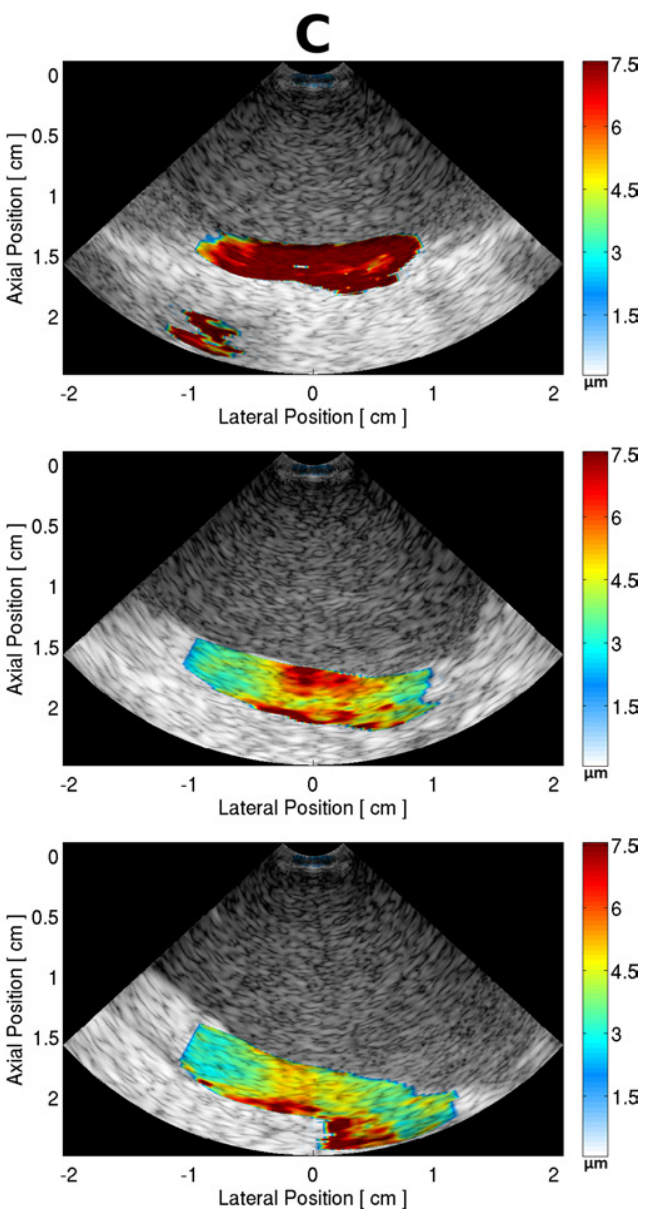

Figure 2 An example of 3 ARFI image-LAT map pairs. Row 1: Acquired before RFA. Row 2: Acquired after intentionally creating a 1-cm conductive gap. Row 3: Acquired after closure of the 1-cm gap. Column A: LAT maps showing the imaging fan position in the canine RA and the location of delivered RFA lesions (red spherical markers). Column B: Conventional B-mode images acquired at the location indicated on the LAT maps. There is no lesion visible in the B-mode images. Column C: Maximum ARFI-induced displacement images. The color bar units are microns of tissue displacement away from the transducer. RFA lesions sites are visible as regions of lower relative displacement (stiffer tissue), represented by the blue-green-yellow portion of the color bar. ARFI = acoustic radiation force impulse; LAT = local activation time; RA, right atrium; RFA = radiofrequency ablation.

\section{Distinguishing conductive from nonconductive unablated gaps}

Cardiomyocytes near sites of RFA delivery can be reversibly "stunned" so that conduction block is observed acutely after circumferential or antral pulmonary vein isolation only to recover at some later time; the reconnection of the pulmonary veins is believed to be an important factor limiting the efficacy of TCA for atrial fibrillation. ${ }^{21-23}$ The discordance between the activation-defined completeness of conduction block and the ARFI imaging-defined lesion contiguity described here could potentially be explained by the indirect functional vs direct imaging method of assessing block. Further investigation will be required to show that imaging tissue stiffness is a more sensitive measure of ablation lesion durability and long-term ablation success than acute activation mapping.

In addition to electrical stunning, edema occurs within RFA-treated regions. ${ }^{24}$ Edema can stiffen the myocardium, and it is unclear whether ARFI imaging is able to differentiate stiffening due to edema from RF-induced coagulation necrosis of the cardiac tissue. This study indicated that
ARFI imaging could detect lesion line discontinuities, but further study of the change in stiffness due to substantial fluid swelling in conductive gaps between RFA lesions is needed.

\section{Study limitations}

The gold standard for image validation is the examination of the tissue pathology. Unfortunately, manually matching the exact orientation and location of each 2D ARFI imaging plane to the ex vivo pathology would risk substantial misregistration error that would affect the accuracy of the lesion evaluations. EAM is the current end-procedure gold standard for assessing ablation line completeness; therefore, this study used EAM to identify regions of conduction disturbance/block at the exact imaging plane as a functional alternative to pathological inspection. The main limitation of using EAM as the gold standard for lesion comparison is that LAT-map conduction disruptions could ultimately only infer, not confirm, the presence of lesions. Also, EA maps are representative geometries of the cardiac chamber. The reproducibility and fidelity of the EA map to the actual 

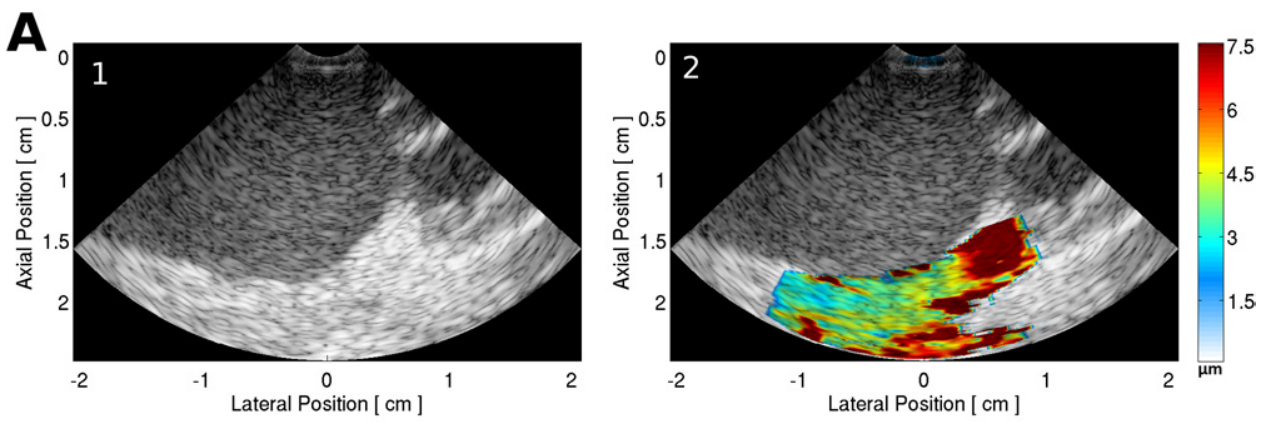

B
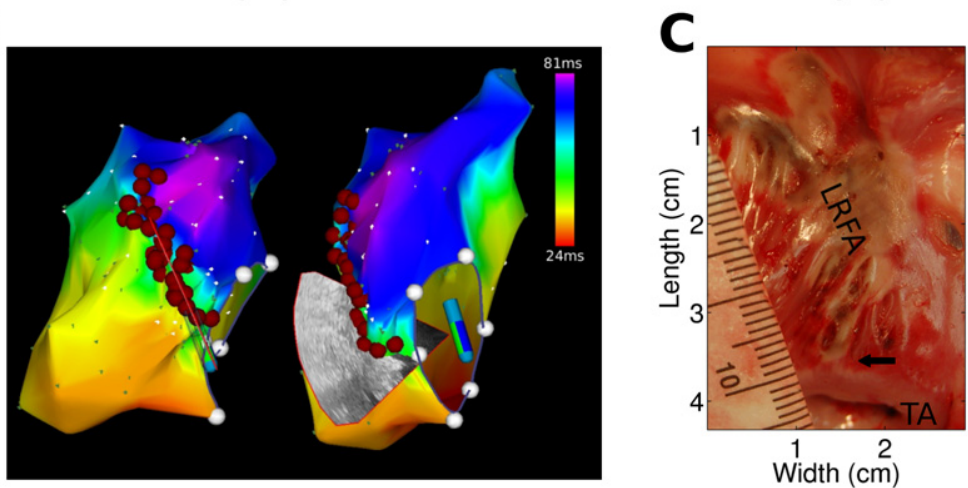

Figure 3 A: Conventional (1) B-mode and (2) ARFI image at the tricuspid annulus (TA). The ARFI image shows an area of relatively high displacements at the annulus, indicating a likely unablated region. ARFI image color bar units are microns displacement away from the transducer. B: LAT map surrounding a line of RFA lesions in the RA; red spheres indicate RFA delivery sites. C: Pathology photograph of the RA endocardial surface confirming continuous line of RFA lesions (LRFA). A small unablated gap is visible at the TA, indicated by the black arrow. ARFI $=$ acoustic radiation force impulse; LAT $=$ local activation time; RA, right atrium; RFA = radiofrequency ablation.

anatomy and electrophysiology is largely dependent on various factors such as catheter stability, LAT-point acquisition density, and respiratory and cardiac motion. For example, Figure $3 \mathrm{C}$ shows an RFA line that was incompletely anchored at the tricuspid annulus. LAT-conduction mapping at the annulus was sparse because of the difficulty of achieving stable catheter contact at the annulus. In this case, it was challenging to verify or deny an anchored lesion line from the LAT map. The ARFI image (Figure 3A) conclusively visualizes an unablated region at the tricuspid annulus that likely corresponds to the unanchored gap. Adding differential pacing to the methods may have improved the accuracy of these ambiguous EAM maps, but this was difficult to achieve in the small canine right atrium and was not performed in this study.

Assessing whether the ARFI imaging plane was parallel and completely transecting a line of conduction block on the LAT map was challenging; the difficulty was reflected in the moderate interreviewer agreement. LAT maps could not provide information about lesion line thickness or irregular lesion morphology if the catheter drifted, making it difficult to predict the exact extent of RFA treatment based on the functional conduction map alone. Misalignment of the imaging plane through complete RFA lines and the corresponding alignment interpretation from the LAT maps was likely a primary source of discrepancy between the ARFI imaging and EAM lesion assessments.

\section{Conclusions}

This study demonstrated that intracardiac ARFI imaging shows promise for evaluating RFA lesions during TCA. Reviewers of the ARFI images successfully identified RFA treatment and potential lesion line discontinuities. Further development of ARFI-specific technology could increase the range of possible imaging depths, allow the imaging of oblique surfaces, and reduce the susceptibility of the images to motion artifacts. Successful clinical implementation of this technology could prompt operators to direct RF energy to incompletely ablated regions, thereby enhancing lesion durability and reducing the need for repeat procedures.

\section{Acknowledgments}

We thank Siemens Healthcare and Biosense Webster for their hardware system and support. We also thank Joshua Hirsch, Stephen Hsu, Brittany Potter, Peter Hollender, Matt Brown, Veronica Rotemberg, Ellen Dixon-Tulloch, and Mark Palmeri for their technical assistance.

\section{References}

1. Calkins H, Kuck KH, Cappato R, et al. 2012 HRS/EHRA/ECAS expert consensus statement on catheter and surgical ablation of atrial fibrillation: recommendations for patient selection, procedural techniques, patient management and follow-up, definitions, endpoints, and research trial design. Heart Rhythm 2012; 9:632-696.e621.

2. Melby SJ, Lee AM, Zierer A, et al. Atrial fibrillation propagates through gaps in ablation lines: implications for ablative treatment of atrial fibrillation. Heart Rhythm 2008;5:1296-1301

3. Kowalski M, Grimes MM, Perez FJ, et al. Histopathological characterization of chronic radiofrequency ablation lesions for pulmonary vein isolation. J Am Coll Cardiol 2012;59:930-938. 
4. Robinson MR, Hutchinson MD. Use of imaging techniques to guide catheter ablation procedures. Curr Cardiol Rep 2010;12:374-381.

5. Nightingale K, Soo MS, Nightingale R, Trahey G. Acoustic radiation force impulse imaging: in vivo demonstration of clinical feasibility. Ultrasound Med Biol 2002;28:227-235.

6. Pinton GF, Dahl JJ, Trahey GE. Rapid tracking of small displacements with ultrasound. IEEE Trans Ultrason Ferroelectr Freq Control 2006;53:1103-1117.

7. Hsu SJ, Bouchard RR, Dumont DM, Ong CW, Wolf PD, Trahey GE. Novel acoustic radiation force impulse imaging methods for visualization of rapidly moving tissue. Ultrason Imaging 2009;31:183-200.

8. Nightingale K, Bentley R, Trahey G. Observations of tissue response to acoustic radiation force: opportunities for imaging. Ultrason Imaging 2002;24:129-138.

9. Palmeri ML, Sharma AC, Bouchard RR, Nightingale RW, Nightingale KR. A finite-element method model of soft tissue response to impulsive acoustic radiation force. IEEE Trans Ultrason Ferroelectr Freq Control 2005;52:1699-1712.

10. Nath S, Redick JA, Whayne JG, Haines DE. Ultrastructural observations in the myocardium beyond the region of acute coagulation necrosis following radiofrequency catheter ablation. J Cardiovasc Electrophysiol 1994;5:838-845.

11. Bosman S, Pickering JW, van Marle J, van Gemert MJ. Ultrastructural alterations in heated canine myocardium. Lasers Surg Med 1995;17:39-48.

12. Pernot M, Mace E, Dubois R, Couade M, Fink M, Tanter M. Mapping myocardial elasticity changes after RF-ablation using supersonic shear imaging. Comput Cardiol 2009;2009:793-796.

13. Fahey BJ, Nightingale KR, McAleavey SA, Palmeri ML, Wolf PD, Trahey GE. Acoustic radiation force impulse imaging of myocardial radiofrequency ablation: initial in vivo results. IEEE Trans Ultrason Ferroelectr Freq Control 2005;52:631-641.

14. Hsu SJ, Fahey BJ, Dumont DM, Wolf PD, Trahey GE. Challenges and implementation of radiation-force imaging with an intracardiac ultrasound transducer. IEEE Trans Ultrason Ferroelectr Freq Control 2007;54:996-1009.
15. Eyerly SA, Hsu SJ, Agashe SH, Trahey GE, Li Y, Wolf PD. An in vitro assessment of acoustic radiation force impulse imaging for visualizing cardiac radiofrequency ablation lesions. J Cardiovasc Electrophysiol 2010;21: 557-563.

16. National Research Council. Guide for the Care and Use of Laboratory Animals. 8th ed. Washington, DC: The National Academies Press; 2011.

17. Nelson TR, Fowlkes JB, Abramowicz JS, Church CC. Ultrasound biosafety considerations for the practicing sonographer and sonologist. J Ultrasound Med 2009;28:139-150.

18. Hsu SJ, Bouchard RR, Dumont DM, Wolf PD, Trahey GE. In vivo assessment of myocardial stiffness with acoustic radiation force impulse imaging. Ultrasound Med Biol 2007;33:1706-1719.

19. Posner KL, Sampson PD, Caplan RA, Ward RJ, Cheney FW. Measuring interrater reliability among multiple raters: an example of methods for nominal data. Statist Med 1990;9:1103-1115.

20. Zar JH. Heterogeneity testing of $2 \times 2$ tables. In: Biostatistical Analysis. Englewood Cliffs, NJ: Prentice-Hall; 1974:62-65.

21. Nath S, Whayne JG, Kaul S, Goodman NC, Jayaweera AR, Haines DE. Effects of radiofrequency catheter ablation on regional myocardial blood flow: possible mechanism for late electrophysiological outcome. Circulation 1994;89:2667-2672.

22. Tai YT, Lee KL, Lau CP. Catheter induced mechanical stunning of accessory pathway conduction: useful guide to successful transcatheter ablation of accessory pathways. Pacing Clin Electrophysiol 1994;17:31-36.

23. Rostock T, O'Neill MD, Sanders P, et al. Characterization of conduction recovery across left atrial linear lesions in patients with paroxysmal and persistent atrial fibrillation. J Cardiovasc Electrophysiol 2006;17:1106-1111.

24. Schwartzman D, Ren JF, Devine WA, Callans DJ. Cardiac swelling associated with linear radiofrequency ablation in the atrium. J Interv Card Electrophysiol 2001;5:159-166. 